Funding Netherlands Epilepsy Foundation (NEF) grant nr 03.18, University Medical Center Utrecht [NL] - institutional Genvlag grant.

\section{PREVALENCE AND CLASSIFICATION OF EPILEPSY AMONG CHILDREN IN A RURAL TEACHING HOSPITAL OF WESTERN INDIA}

doi:10.1136/archdischild-2012-302724.1509

${ }^{1} \mathrm{NH}$ Thacker, 'L Dalal, 'NM Kharod, 1,2SM Nimbalkar. 'Department of Pediatrics, Pramukhswami Medical College; ${ }^{2}$ Central Research Services, Charutar Arogya Mandal, Anand, India

Background and Aims To study Prevalence of Active Epilepsy in children and Clinical pattern of seizures among epileptics. Classification of epilepsy and epileptic syndromes was done according to ICEES,1989.

Methods All 0-18 year old patients; visiting Hospital during the study period. The ones with Active Epilepsy were studied for clinical pattern of seizures and classification of epilepsy.

Results 4981 patients visited the hospital which included 2893 (58\%) males and 2088 (42\%) females. Active Epilepsy was found in 41 boys and 19 girls. Crude Prevalence was 12 per 1000. Gender specific prevalence was higher in males $14.1 / 1000$ as compared to females 9.1/1000. Age specific prevalence was maximum 19.2/1000 in age group of 5-10 yrs. Maximum patients (25\%) had their seizure debut in First year of life. Secondarily generalized seizures were the most common type of seizures with 20 (33.3\%) of patients having it as the main seizure type. Wests syndrome and Lennox Gastaut syndrome were the most common generalized epileptic syndromes. $20 \%$ had Idiopathic Epilepsy, 30\% probably Symptomatic (earlier Cryptogenic) and $50 \%$ had Symptomatic epilepsy. Perinatal asphyxia accounted for $36.7 \%$ of symptomatic epilepsies. $43.3 \%$ of patients had neurohandicaps of which CP alone accounted for $53.8 \%$. $31.7 \%$ of the patients were on multiple AEDs, $30 \%$ were on Valproate alone at the time of presentation.

Conclusions This study emphasizes the fact that perinatal asphyxia, CNS infections are major contributing factors to childhood epilepsy also leading to a higher percentage of Wests syndromes and LG syndrome which is not the case in western studies.

\section{A STUDY OF THE VALUE OF SLEEP EEG RECORD IN PREDICTING SEIZURE RECURRENCE IN CHILDREN WITH A SINGLE AFEBRILE SEIZURE}

doi:10.1136/archdischild-2012-302724.1510

1,2J Chukwu, ${ }^{3 P}$ Gallagher, ${ }^{3} \mathrm{~B}$ McCoy, ${ }^{3} \mathrm{D}$ Webb. 'Molecular \& Cellular Therapeutics, Royal College of Surgeons; ${ }^{2}$ Clinical Research Unit, National Children's Research Centre; ${ }^{3}$ Neurology, Our Lady's Children's Hospital, Dublin, Ireland

Aims The main aim of the study was to determine the value of a sleep EEG recording in predicting the risk of epilepsy in children with a single afebrile seizure.

Methods A retrospective review of 69 children who underwent sleep EEG recordings in 2007 following their first afebrile seizure was undertaken. Parents were sent a questionnaire on seizure recurrence, diagnosis of epilepsy, anti-epileptic medication use, further EEGs and school progress.

Results Fifty two (52) questionnaires (75\%) were completed and returned. Twenty three of the fifty two children (44\%) have had one or more further seizures $-7 / 25(28 \%)$ of those with normal sleep EEG recordings and 14/21 (67\%) of those who had abnormal sleep EEG recordings. 6/52 (12\%) of the respondents had suspicious EEG and 2 of these have had further seizures. 12/21 (57\%) of those with abnormal sleep EEGs compared with 5/25(20\%) of those with normal EEG have had multiple further seizures.
Pearson-Chi Square revealed a significant relationship between abnormal EEG and the development of further seizures $(p<0.01)$. The odds of having further seizures if the EEG was abnormal was 4.9 (95\% C.I 1.5-16.1).

Conclusions Sleep EEG is a useful tool in predicting the risk of developing epilepsy in children with first afebrile seizure.

\section{DOES NEPHROTOXICITY EXIST IN EPILEPTIC PATIENTS ON VALPROATE OR CARBAMAZEPINE THERAPY ? (A PRELIMINARY STUDY)}

doi:10.1136/archdischild-2012-302724.1511

${ }^{1} \mathrm{C}$ Havali, ${ }^{1} \mathrm{~K}$ Gücüyener, ${ }^{2} \mathrm{~N}$ Buyan, 'E Gürkaş, 'E Demir, 'A Serdaroğlu. 'Pediatric Neurology; ${ }^{2}$ Pediatric Nephrology, Gazi University Faculty of Medicine, Ankara, Turkey

Objective The aim of the study was to investigate renal glomerular and tubular side effects of valproate and carbamazepine if exists, in children who are on these antiepileptic drugs between 6 months and two years of time.

Method A prospective study was performed on epileptic children (primary generalized and partial epilepsy) under valproate (n:30), carbamazepine (n:24) treatments and healty control group (n:26). The serum creatinine, Cystatin $C$ levels and urinary excretion of $\mathrm{N}$-acetyl- $\beta$-D-glucosaminidase levels were taken at the beginning of the study and after 6 months of therapy. Three Glomerular filtration rate formulae with creatinine, Cystatin $\mathrm{C}$ and combined of all of were used to determine glomerular filtration functions.

Results Serum creatinine, Cystatin C levels of patients and glomerular filtration rate values were in the normal range according to patient ages and healthy control group. However urinary $\mathrm{N}$-acety$\beta$-D-glucosaminidase/creatinine levels were higher in both groups (valproate 6.1 \pm 5 ) (carbamazepine 3.14 \pm 1.97 ) when compared to the levels of the control group $(2.6 \pm 1.3)(p<0.05)$ Although this is a preliminary study; bearing in mind that patients on both antiepileptic drugs may have a tubulotoxicity risk; renal function test should also be checked in during the treatment of all patients with special emphasis when there is a renal or systemic disease co-existing.

\section{VALIDITY OF THE CLINICAL NEUROLOGICAL STATE IN DIAGNOSING DIABETIC PERIPHERAL NEUROPATHY}

doi:10.1136/archdischild-2012-302724.1512

1,21 Hoeliner, 'V Haslinger, 'J Lütschg, 'G Müller, 'D Seick, ${ }^{3} \mathrm{~J}$ Fussenegger, ${ }^{3} \mathrm{U}$ Zanier, ${ }^{2,4} \mathrm{C}$ Saely, ${ }^{2,4} \mathrm{H}$ Drexel, 'B Simma. 'Paediatrics, Academic Teaching Hospital, Feldkirch, Austria; '2Private University of the Principality of Liechtenstein, Triesen, Liechtenstein; ${ }^{3}$ Krankenhaus Dornbirn, Dornbirn; ${ }^{4}$ Vorarlberg Institute for Vascular Investigation and Treatment, Feldkirch, Austria

Objective We aimed to evaluate the prevalence of diabetic peripheral neuropathy (DPN) in children and adolescents with type 1 diabetes mellitus (T1DM) and examine whether the clinical neurological state validly diagnoses DPN as compared to the gold standard of nerve conduction velocity (NCV) in these patients.

Methods We measured NCV in an unselected consecutive series of patients aged 8-18 years who had been suffering from T1DM for at least one year. The clinical neurological state of these patients was examined using a protocol including ankle reflex, vibration, pinprick, and temperature testing as well as a standardized questionnaire; neuropathy disability scores (NDS) and neuropathy symptom scores (NSS) were assessed.

Results Of our 39 patients, six (15\%) had clinically evident DPN, whereas NCV testing revealed DPN in 15 (38\%) patients. Sensitivity and specificity of the clinical neurological exam for the diagnosis of DPN as compared to the gold standard of pathological NCV were $40 \%$ and $100 \%$, respectively. The corresponding positive and negative predictive values were $100 \%$ and $72.7 \%$, respectively. 